\title{
Determination of the Location of Stomach Polyps from Endoscopic Examination Using Chan-Vese Segmentation Which is an Image Processing Technique
}

\author{
[ Ali YASAR, Ismail SARITAS ]
}

\begin{abstract}
Stomach polyps are sessile or stalked lesions originating from stomach epithelial or submucosa and protruding to lumen.Observation rate of stomach polys during endoscopic procedures is $\mathbf{2 - 6 \%}$. It is known that these are asmptomatic and also cause bleeding anemia, abdominal pain gstricoutlet obstruction. The most common polyps in the stomach are reported as hyperplastic polyps and hazelnuts gland polyps. In this study, a system has been designed for the detection of stomach polyps using image procesing techniques from the endoscopy images of Gastroenterology clinic patients in Selcuk University Faculty of Medicine Hospital.
\end{abstract}

Keywords - Stomach Polyp, Image Processing, Chan-Vese Segmentation, Gastrit Polyp

\section{Introduction}

Stomach polyps are more common in adults and they are single or multiple benign tumors. Polyps localized in the distal part of the stomach gives moe syptoms. Wen the polyps in the stomach are diagnosed, the cancer should be ruled out.

Stomach polyps are istologically classified as hyperplasti, adenomatous or inflamatory. Polypoid lesions such as leiomyomas and carcinoid tumors have been discussed in other threads. Hyperplastic olyps whch comprises $80 \%$ of the cases are caused by the overgrowth of normalepithelium.

Approximately $30 \%$ of adenomatous polyps contain adenocarcinoma focus. Also \%20 of adenocarcinoma can be found somewhere else in the stomach of the patients which has benign adenomatous polyps.

\section{Ali YASAR}

Computer Programming, Guneysinir Vocational School of Higher Education Selcuk University

Turkey

Ismail SARITAS

Department of Electronic Engineering, Technology Faculty, Selcuk University

Turkey
The incidence of cacer in adenomatou plyps increases with the size growth of the poly. Lesions with handle and diameter that are smaller than $2 \mathrm{~cm}$ are not usually malignant. Approximately $\% 10$ of benign adenomatous polyps change in the direction of malignancy during their development[1].For these resons early detection is important. Therefore, studies in edical image processing need safe and effective image segmentation. Segmentation process is of paramount importance for the extraction of results in image processing. In our study, Chen-vesa segmentation method has bee used. Because Chan-Vese method is a powerful and flexible geometric active contour model that can detect objects whose boundaries are not necessarily marked with a gradient. Similar to other active contour models, it aims an energy function to be minimized. Unlike others, its stopping term has not a dependency on image gradient, however. For this reason, it can achieve the segmentation of tissue structures, some of which would be difficult to define object boundaries due to the complexity of the tissue structures. The aim of this stud $s$ to perform a classification process for the endoscopy images that we have with Chan-Vese segmentation.

\section{Material ve Methods}

Depending on the applications of medical image processing, removal of a significant portion of the image is the most important diagnostic step. This process is called "segmentation" in literature. In other words, segmentation is a key step in image definition and segmentation. Portion of the image obtained as a result of image segmentation is called "relevant area"[2]. In literature, many different methods have been used for segmentation process. Multiple gray level thresholding and rules-based approaches have been used for nodule detection while Lo and colleagues have proposed a two-tier system [3-4].

Accordingly, possible examples of nodules has been placed in the first stage, while in the second stage, nodular ones and the not nodular ones were separated using cellular artificial neural network (CANN). Another study following the detection of nodules using $\mathrm{ANN}$ realized by $\mathrm{Xu}$ and colleagues includes a set of forward orientation ANN and decision rules. In our study, Matlab R2015a Image Processing and Computer Vision toolbox has been used [5]. In our study, Matlab R2015a Image Processing and Computer Vision toolbox is used. 


\section{A. Regional Based Active Credit Method}

Image segmentation is a basic image processing operation used to distinguish objects within the image. One method of image segmentation method which was proposed by Chen-Vesa is the use of active credit and level set[6]. In level set theory, the curve pulls its lines to the desired edge and boundaries during the evolution. On the other hand, in active credit method, the first ideas is to use a deformable model and to minimize the energy function for each iteration. For active credit, two methods are used which are edge based and region based. While an edge detector is used for edge based method, detection of background and foreground is used for region based method[7]. In many cases asmooth segmentation can be realized for edge based method due to the basence of a global limiting on the image. On the other hand, region based method is adventageous than edge based method, because the first placed curve is solid and insensitive to the noise. However, the disadvantage of region based method is that it leads to false image segmentation occasionally. The basic idea of this method is that the closed curve (credit) achieve results starting with the first mask (a square shape, etc.) and then through expansion or contraction based on image restrictions. This process is done by minimizing the energy function and is known as the evolution of credit. Active credit formulation is based on two-dimensional Lipschitz function defined in the image plane. his function is called level set function. Initial level set function is named a signal distance function (SDF)[8]. Level set function $\varphi=(\mathrm{x}, \mathrm{y})$ is a two-dimensional Lipschitz function and it is applied for showing the credit values. Also, closed credit $\mathrm{C}$ curvecan be shown indirectly by the zero level in $\varphi^{\prime}$

Credit can be pozitive or negative or it can be between the two. The credit development can be expressed with the equation of $\frac{\partial c}{\partial t}=\frac{\partial \varphi(x, y)}{\partial t}$. If $\varphi(x, y)=0, C 1$ express a point on the curve. If $\varphi(x, y)>0, C 3$ express the outside of the curve and if $\varphi(\mathrm{x}, \mathrm{y})<0, \mathrm{C} 2$ express the inside of the curve.

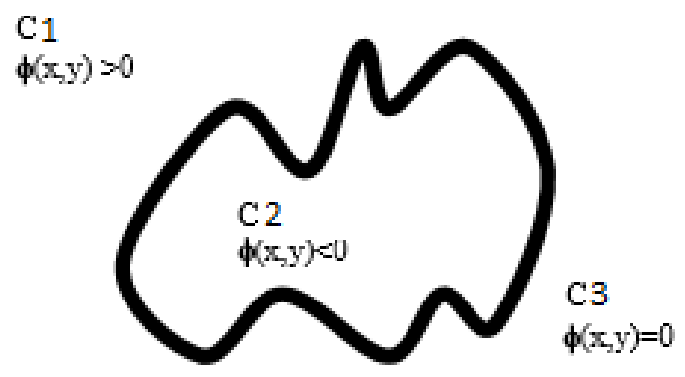

Fig. 1: Credit Development

Heaviside function is used to prevent sudden transitions from zero to 1 or 1 to zero on $\mathrm{C}$ curve. Therefore, the system output is guaranteed to be stable. Also, the outside area is expressed by $(1-H(\varphi))$

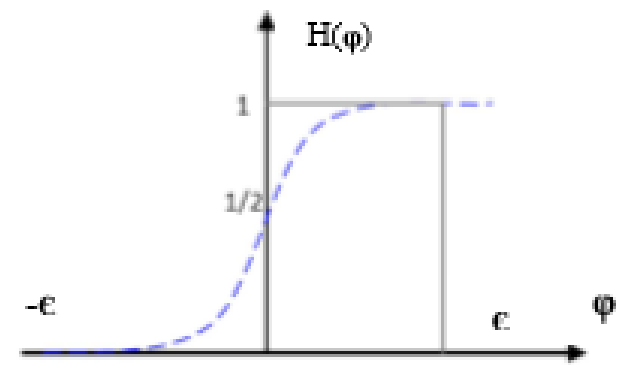

Fig. 2: Heaviside Function

$$
H(\varphi)=\left\{\begin{array}{cc}
1 & \varphi>\epsilon \\
\frac{1}{2}\left(1+\frac{\varphi}{\epsilon}+\frac{1}{\pi} \sin \left(\frac{\pi \varphi}{\epsilon}\right)\right) & |\varphi|<\epsilon \\
0 & \varphi<-\epsilon
\end{array}\right\}
$$

The energy function can be shown as equation 3 .

$$
\begin{aligned}
F= & \mu \cdot u z u n l u k(C)+v \cdot A l a n(i c i(C)) \\
& +\lambda_{1} \int_{i c i c}|I(x, y)-u|^{2} d x d y \\
& +\lambda_{2} \int_{d i s y c C}|I(x, y)-v|^{2} d x d y
\end{aligned}
$$

In equation 3, I express the image and $\mathrm{u}$ and $\mathrm{v}$ are constants related to $\mathrm{C}$. Also, $\mu, \lambda 1$ and $\lambda 2$ are positive constants. For numerical calculations, $\lambda 1$ and $\lambda 2$ are set to 1 and $\mathrm{v}$ is set to 0 . Taking into account the minimization problem, to minimize the energy function according to $\mathrm{v}$ and $\mathrm{y}, \varphi$ is fixed. Thus, $\mathrm{u}$ and $\mathrm{v}$ related to $\varphi$ can be shown as following.

$$
\begin{aligned}
& u=\frac{\int_{\Omega} I(x, y) H(\varphi(x, y)) d x d y}{\int_{\Omega} H(\varphi(x, y)) d x d y} \\
& v=\frac{\int_{\Omega} I(x, y)(1-H(\varphi(x, y))) d x d y}{\int_{\Omega}(1-H(\varphi(x, y))) d x d y}
\end{aligned}
$$

Here, $\mathrm{u}$ and $\mathrm{v}$ can be indicated as inside and outside areas. To update the curve, the level set function has to be restarted. The restarted function can be shown as in equation 5 .

$\varphi^{t+1}=\varphi^{t}-\Delta t \cdot S\left(\varphi_{0}\right) \cdot G(\varphi)$

Zero level set limits the calculation with a narrow band around. The structure in Fig. 3 is named as narrow band. When zero level set approaches to the edge of the narrow band, the level set function is restarted. The narrow band is recalculated. Restart is not required while $\varphi$ is calculated for the whole image. Because restart does not cause an effective 
Proc. of the Third Intl. Conf. on Advances in Bio-Informatics and Environmental Engineering - ICABEE 2015 Copyright ( $($ Institute of Research Engineers and Doctors, USA .All rights reserved.

ISBN: 978-1-63248-078-1 doi: 10.15224/ 978-1-63248-078-1-117

impact on the result while $\varphi$ is calculated. Therefore, restart can be negligible. [9]

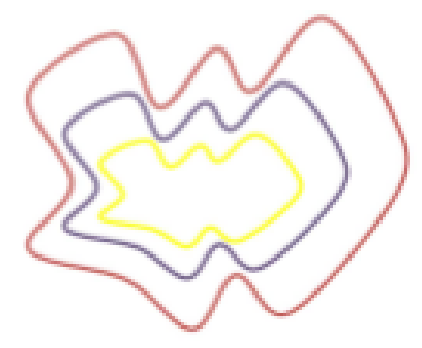

Fig. 3: Narrow Band innermost curve represents the zero level set.

\section{Result and Discussion}

Endoscopy images belonging t the patient are shown in Fig. 1(a), Fig. 2(a) and Fig. 3(a). The applications of Chan-Vese segmentation are shown in Fig. 1(b) Fig. 2(b) and Fig. 3(b) and Masked images of Chan-Vese segmentation process results are shown in Fig. 1(c), Fig. 2(c) and Fig. 3(c).

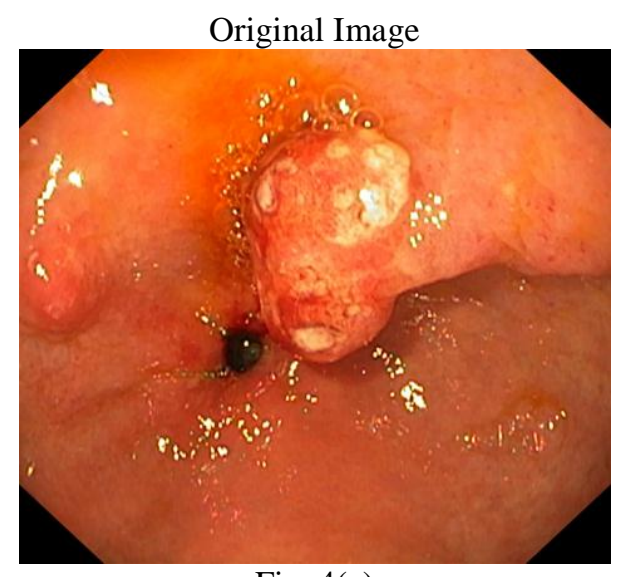

Fig. 4(a)

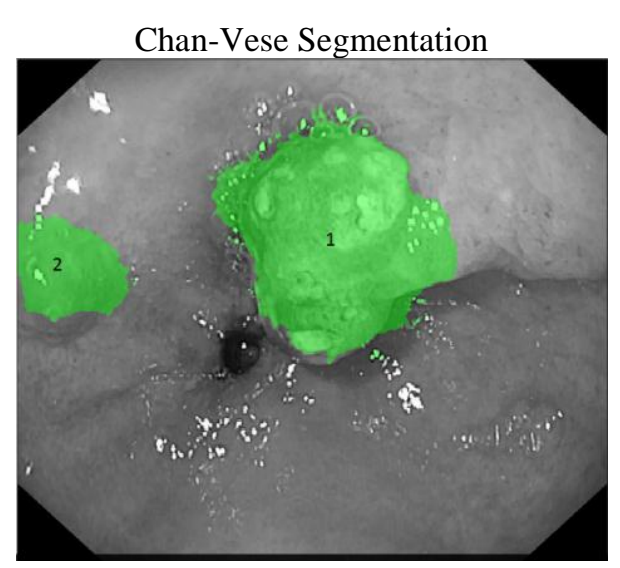

Fig. 4(b)

Masked Image

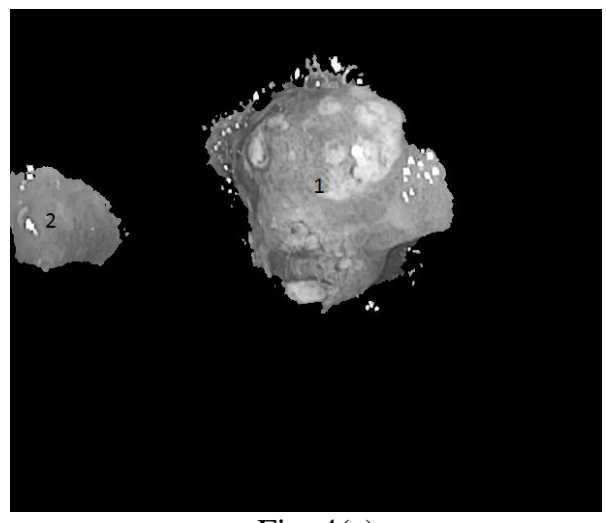

Fig. 4(c)

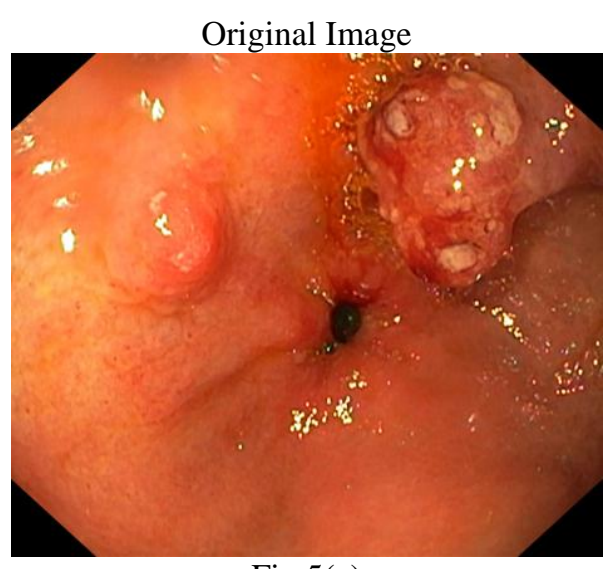

Fig.5(a)

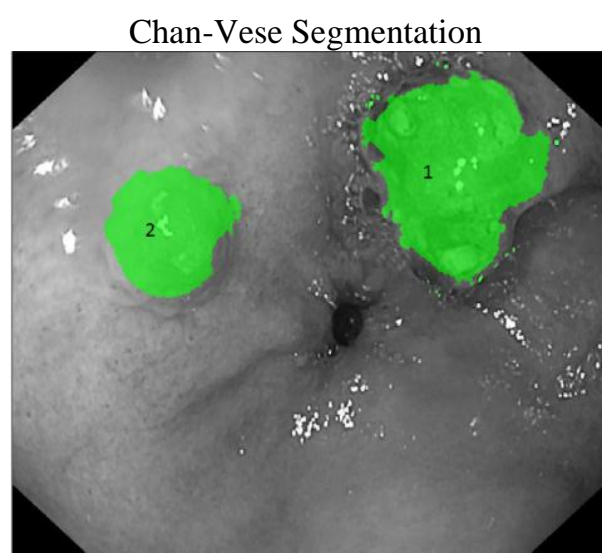

Fig. 5(b)

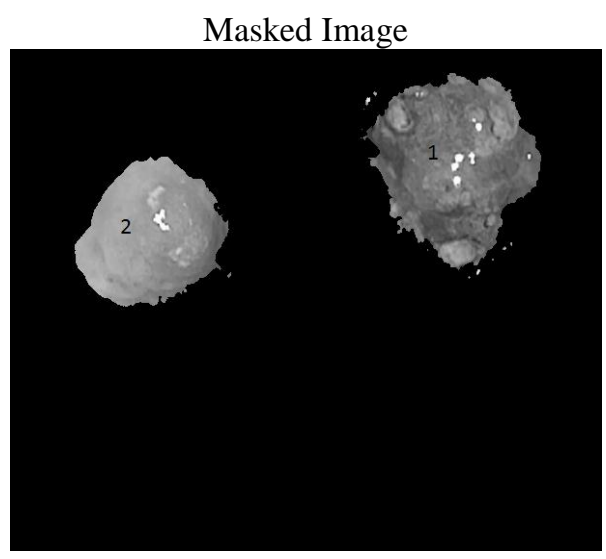

Fig. 5(c) 
Proc. of the Third Intl. Conf. on Advances in Bio-Informatics and Environmental Engineering - ICABEE 2015

Copyright (C) Institute of Research Engineers and Doctors, USA .All rights reserved.

ISBN: 978-1-63248-078-1 doi: 10.15224/ 978-1-63248-078-1-117

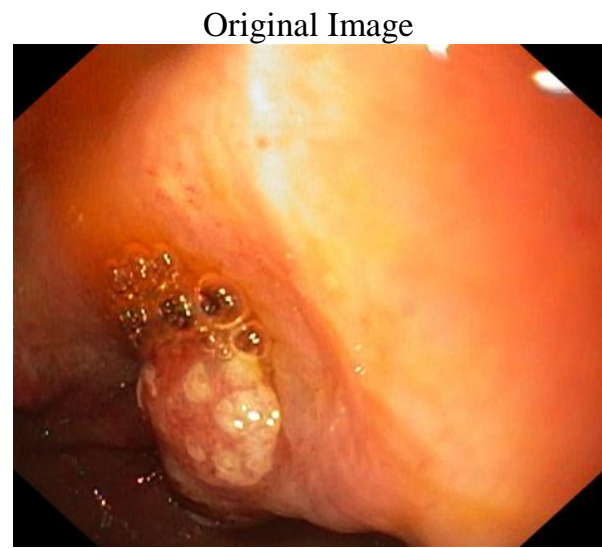

Fig. 6(a)

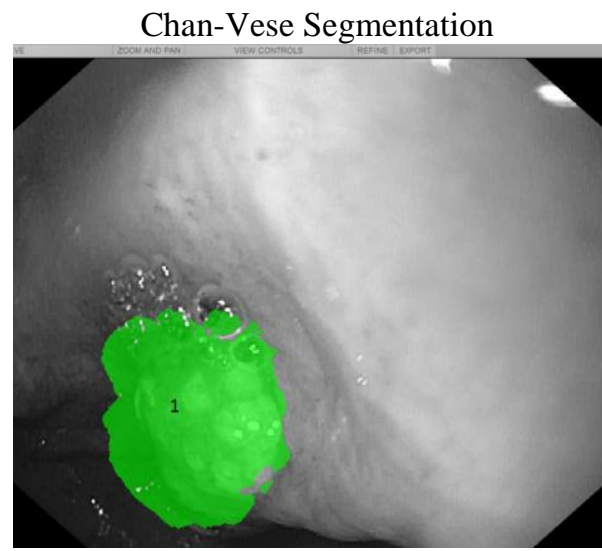

Fig. 6(b)

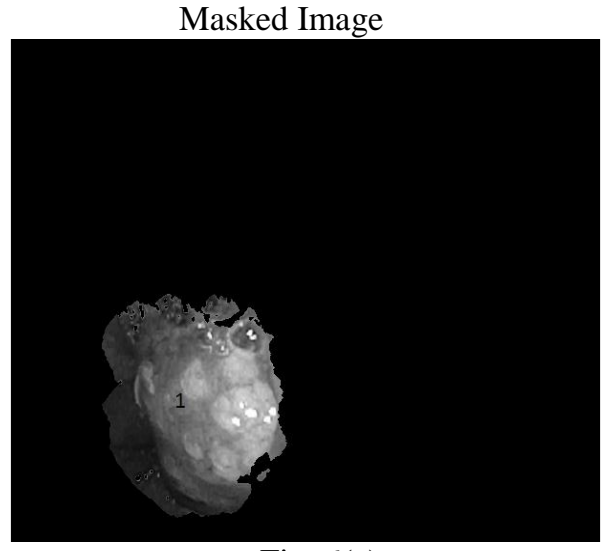

Fig. 6(c)

According to the masking method applied based on ChanVese segmentation method in image processing, during the evaluation of the performance of detecting polyps, success performance of the indicated polyp area by the doctor and the polyp are resulting from our study is shown in Table 1.

TABLE I. POLYP ARE RESULTING

\begin{tabular}{|l|c|c|c|c|c|}
\hline \multicolumn{7}{|c|}{ Total Pixel Count / The number of pixels produced } \\
\hline & $\begin{array}{c}\text { Image } \\
\text { 1(1) }\end{array}$ & $\begin{array}{c}\text { Image } \\
\text { 1(2) }\end{array}$ & $\begin{array}{c}\text { Image } \\
\text { 2(1) }\end{array}$ & $\begin{array}{c}\text { Image } \\
\text { 2(2) }\end{array}$ & $\begin{array}{c}\text { Image } \\
\text { 3(1) }\end{array}$ \\
\hline Expert & 28690 & 14414 & 47485 & 9216 & 42000 \\
\hline Study & 32542 & 16328 & 50592 & 11863 & 26988 \\
\hline
\end{tabular}

As a result of the comparison between the polyps determined by the doctor and resulted from our study, it seems that we achieved a successful outcome of \%88 from Fig. 4(1), \% 88 for Fig 4(2), \%93,8 for Fig 5(1), \% 77,6 for Fig 5(2) and \%64,2 for Fig 6(1).

\section{Conclusion}

In this study, it is seen that Chan-Vese segmentation method can be used for the determination of stomach polyps and successful results can be obtained. For other studies, different segmentation processes are expected to produce better results. Studies will continue along with this thought.

\section{Acknowledgements}

- Appreciations to the doctors and the staff of Selcuk University, Faculty of Medicine Hospital, Gastroenterology clinic for their support in obtaining pictures necessary for our study. This study has been supported by Selcuk University’s Scientific Research Unit (BAP)

\section{References}

[1] http://www.saglikbilimi.com/mide-polipleri/ Last Connection 02.11.2015

[2] Wismüller, A., F, Vietze., 2000, Segmentation with neural network, Academic Press.

[3] Ginger, M. L., Giger, M.L., Bae, K.T., MacMahon, H., 1994, Computerized detection of pulmonary nodules in computed tomography images, Invest. Radiol. 29, 459-465.

[4] Lo, S.C.B., Lou, S.-L.A., Lin, J.-S., Freedman, M., Chien, M.V., Mun, S.K., 1995, Artificial convolutional neural network techniques and applications for lung nodule detection, IEEE Transactions on Medical Imaging, 14, 711-718.

[5] Xu, X.W., Doi, K., Kobayashi, T., MacMahon, H., Giger, M., 1997, Development of an improved CAD scheme for automated detection of lung nodules in digital chest images, Medical Physics, 24, 1395-1403.

[6] T.Chan, L.Vese, "Active Contours Without Edges", IEEE transactions on image processing, vol.10,no.2,February 2001.

[7] S. Lankton, A. Tannenbaum, "Localizing RegionBased Active Contours" IEEE transactions on image processing,vol.17,no.11 November 2008

[8] C. P. Lee, "Robust Image Segmentation using Active Contours: Level Set Approaches", dissertation, North Carolina State University

[9] TUNCER S.A, ALKAN A. "Troit Nodüllerinin Bölge Tabanli Aktif Şekil (Contour) Yöntemi İle Bölütlenmesi ",2011 